\title{
Right coronary artery territory ischemia after pulmonary artery banding in univentricular physiology with transposition of great arteries
}

\author{
Sachin Mahajan ${ }^{1}$, Sudhansoo Khanna ${ }^{1}$, Sanjeev Naganur ${ }^{1}$, and SHEENAM WALIA ${ }^{1}$ \\ ${ }^{1}$ Post Graduate Institute of Medical Education and Research
}

April 27, 2020

\begin{abstract}
Pulmonary artery (PA) band is done in a variety of congenital heart diseases (CHDs) with the primary goal of reducing pulmonary overcirculation. Its use has declined during the last two decades, however, its role still exists in classic univentricular heart with unrestricted pulmonary blood flow. PA banding in univentricular morphology with transposition of great arteries (TGA) can be extremely morbid. Our patient had ST segment elevation in inferior leads after banding, indicating acute ischemia in right coronary artery territory. We hypothesize that, the cause for this was sudden shift of volume to the rudimentary camber through the bulboventricular foramen after PA banding, causing subendocardial ischemia. Interpretation of electrocardiogram (ECG) and subsequent management is not straightforward in this scenario. We hereby describe successful management of sudden ST segment changes observed in immediate post-operative period after PA banding for univentricular, TGA heart in an 8 month old infant.

\section{Text}

\section{Introduction}

Pulmonary artery (PA) banding is used as a palliative treatment for infants, who are not candidates for immediate primary repair. In patients of single ventricle (SV) physiology with unrestricted blood flow, it is done as a first stage palliation to protect the lungs. PA banding in univentricular situation with transposition of great arteries (TGA), can be extremely morbid. ${ }^{1}$ Sudden onset of increase in afterload and systemic hypoxemia after PA banding, can produce ischemia in a single ventricle. But these should cause global ischemia and not regional, as observed in our patient. We believe that sudden shift of preload to rudimentary chamber after PA banding causes increased wall stress. This leads to subendocardial ischemia and hence ST segment elevation in RCA territory. New onset ischemia in a banded single ventricle, if not managed appropriately, can lead to dismal outcome. The literature highlighting its management is very limited.
\end{abstract}

\section{Case Report}

An eight month old infant was brought to our emergency services with features of failure. A systolic murmur was heard over left sternal border. Chest X-ray revealed cardiomegaly and pulmonary plethora. In view of respiratory distress, she was provided invasive ventilator support. Electrocardiogram (ECG) revealed sinus rhythm, right axis of QRS vector and mild ST depression of inferior leads (figure 1). On trans-thoracic echocardiography (TTE), single ventricle (SV) physiology in the form of double inlet left ventricle (DILV), large bulboventricular foramen and levo transposition of great vessels (l-TGA) was found. Aorta was arising from rudimentary chamber. Recurrent episodes of left lung collapse while on mechanical ventilation, delayed weaning of the child. Decongestive measures were maximized and patient was weaned off from the ventilator 
after 12 days. However, the infant remained in respiratory distress even with supplemental oxygen. In view of intractable failure, PA banding was planned. Preoperatively, patient had saturation $\left(\mathrm{SpO}_{2}\right)$ of $100 \%$ with $\mathrm{FiO}_{2}$ of 0.5 .

Intra-operatively, main pulmonary artery (MPA) was dilated with l-TGA relationship of great vessels. MPA was banded starting with the perimeter of $26 \mathrm{~mm} \mathrm{Hg}$ while monitoring PA pressure distal to the band (Figure 2). With subsequent clipping, mean PA pressure of $10 \mathrm{~mm} \mathrm{Hg}$ with $\mathrm{SpO}_{2}$ of $85 \%$ was achieved. The gradient across the band was $55 \mathrm{~mm} \mathrm{Hg}$ and visual contractility was good. Patient was shifted to ICU with minimal dose of milrinone.

After 3 hours post-operatively, patient developed sudden tachycardia, with ST segment elevation in the inferior leads (Figure 3). Apart from tachycardia, patient was hemodynamically stable. Subtle decompensation in the form of cold extremities, borderline urine output, lowering of saturation and increased lactate were observed. Chest X-ray revealed collapse of the left lung. TTE revealed mild ventricular dysfunction without any atrio-ventricular (AV) valve regurgitation. We never resorted to vasopressors. On the contrary, milrinone was maximized and aggressive diuresis was induced. Positive en expiratory pressure (PEEP) and total volume were regulated. Repeated suctioning and recruitment were done in view of left lung collapse. ST segment reverted back to baseline rhythm after few hours of these therapeutic adjustments (Figure 4). This correlated with gradual improvement in the cardiac output and patient was weaned off successfully.

\section{Discussion}

In 1952, Muller and Dammann described pulmonary artery banding (PAB) for palliation of congenital heart disease (CHD) with pulmonary hypertension (PAH) ${ }^{3}$ As a first step in palliation, PA banding is the only option, for univentricular physiology patients with unrestricted pulmonary blood flow. We believe acute regional ischemia, following PA banding especially in patients with univentricular physiology, has not been addressed adequately in the literature. Our patient had ST segment elevation in inferior leads after 3 hours of PA banding indicating acute ischemia in RCA territory. In majority of patients with DILV, L - TGA, the systemic vessel arises from the rudimentary chamber. We hypothesize that sudden shift of preload to the less compliant rudimentary chamber after PA banding, causes increased wall stress and hence subendocardial ischemia in the RCA territory.

In the first instance, pressure by the band was thought as a possibility, since the great vessels were almost antero-posterior relation. As per the observation of operating surgeon, the band was away from proximal RCA, since RCA arises from the lateral aspect of anterior systemic vessel. Pressure by the band can be a possbilty, if RCA arises posteriorly, making it vulnerable to compression.

Before onset of ischemic changes, there was no hemodynamic aberration apart from tachycardia. Tachycardia itself can lead to elevation of ST segment. However, in that case, it should have been global and not regional, as observed in our case. Rakhi et al reported ST depression in all the electrocardiographic leads soon after PA banding in a similar clinical situation. ${ }^{2}$ These changes persisted despite elevating the systemic pressures with inotropes and fluid replacement. We believe decreased myocardial supply is unlikely to be the cause, as effective PA banding will anyway increase the systemic blood pressure more than the baseline. We maximized milrinone after acute ischemia in our case and we believe it helped. Using indilators, to decrease the ventricular wall stress is more physiological. On the contrary, vasopressors will increase the myocardial oxygen demand and add to afterload, hence can compound ischemia.

Echocardiography done at that time revealed mild ventricular dysfunction and no AV valve regurgitation. Our observation of mild hemodynamic instability inspite of acute ischemia can be explained by the fact that, majority of the dominant ventricle is supplied by left coronary. Mild hemodynamic instability allowed us to pursue aggressive diuresis as well. We believe, by decreasing end diastolic volumes, by diuretics, will decrease wall stress and hence improve subendocardial perfusion.

There is a marked decrease in the pulmonary blood flow after banding, leading to decrease in arterial saturation. This coincides with the increased oxygen demand immediately after banding. Diuretics can improve 
arterial saturation in these high pulmonary blood flow patients, by removing excess water. Applying PEEP and aggressively treating atelectasis can prevent intrapulmonary shunting .Optimization of lung function can substantially mitigate hypoxia, which we believe is an important compounding factor.

To conclude, ischemia after PA banding in univentricular physiology can lead to dismal outcome. Use of vasopressors to increase systemic pressures, will not help. Liberal use of diuretics and inodilators, can shorten phase of acute remodeling and hence limit ischemia. Once equilibrium is achieved, ischemia will resolve in a banded single ventricle.

\section{Author contributions}

Dr. Sachin Mahajan- Concept/ design.

Dr. Sudhansoo Khanna - Data analysis, Drafting article

Dr. Sanjeev Naganur- Critical revision of article.

Dr. Sheenam Walia - Data collection.

\section{References}

1. Sharma R. Pulmonary artery banding: Rationale and possible indications in the current era. Ann Pediatr Cardiol 2012;5(1):40-43.

2. Retnamma RK, Nair SG, Sunil GS, Benedict R. ST segment depression following pulmonary artery banding. Ann Card Anaesth2012;15(1):64-66. doi:10.4103/0971-9784.91486

3. Muller WH Jr, Danimann F Jr. The treatment of certain congenital malformations of the heart by the creation of pulmonic stenosis to reduce pulmonary hypertension and excessive pulmonary blood flow; a preliminary report. Surg Gynecol Obstet 1952;95(2):213-219.

Figure legendsFigure 1: Preoperative ECG showing sinus rhythm, right axis of QRS vector and mild ST depression of inferior leads.Figure 2: Intra-operative photograph showing PA band, RCA.Figure 3: ECG (3 hours postoperatively) showing ST segment elevation in the inferior leads (II, III, aVF).Figure 4: ECG showing mild ST depression of inferior leads, back to baseline rhythm after therapeutic maneuvers.

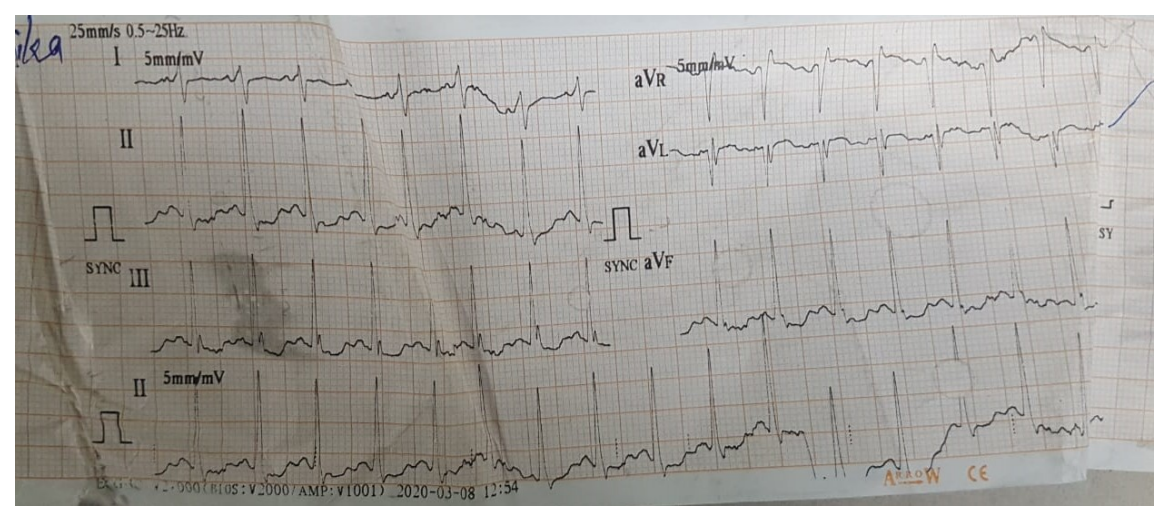



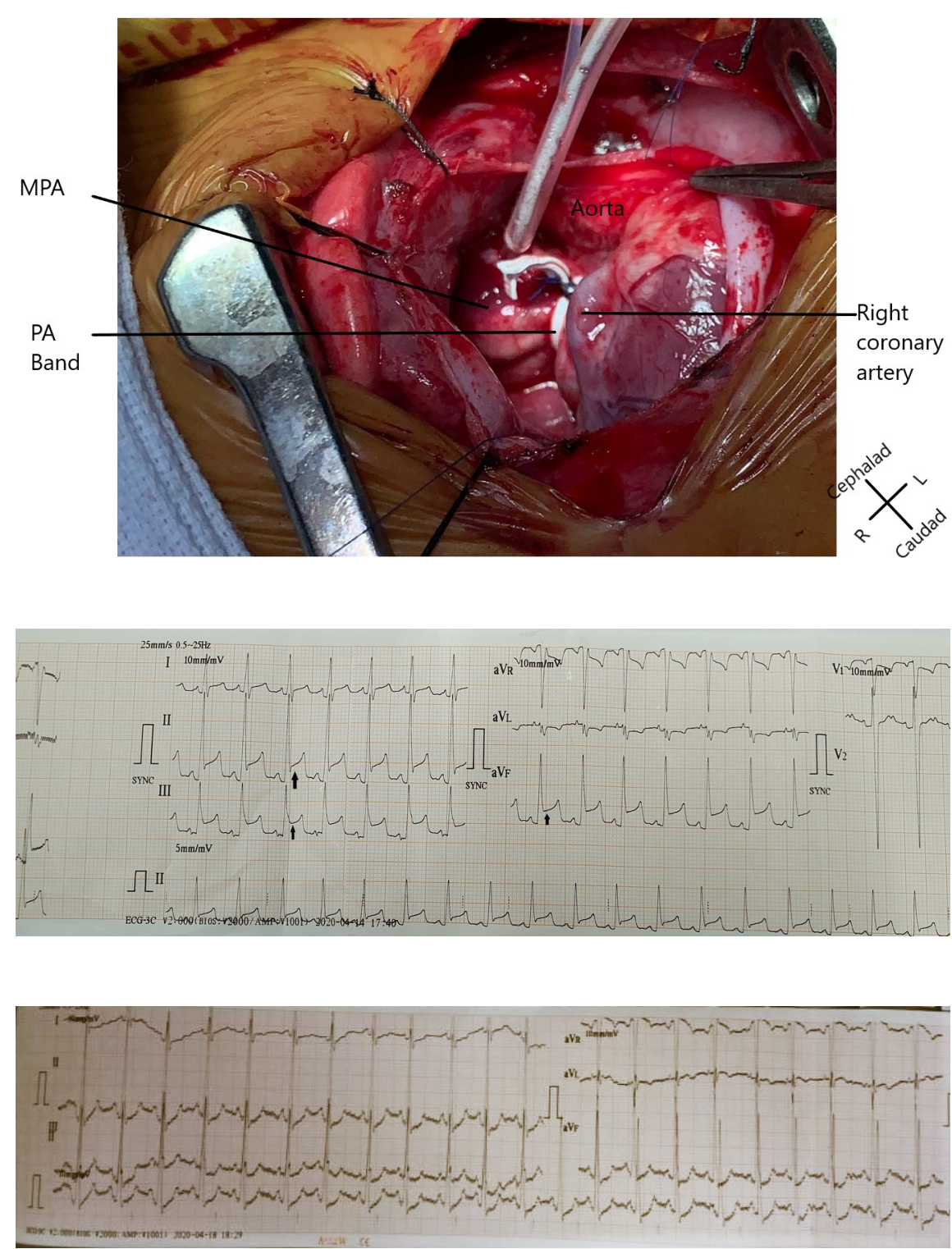